\title{
Test yourself answer: exophytic slow-growing foot lesion
}

\author{
Fabiana Migliaccio Mansur ${ }^{1}\left[\right.$ ] Tullia Cuzzi $^{2}$ - Marcelo Bragança dos Reis Oliveira ${ }^{3}$ Flávia Martins Costa ${ }^{1,4}$. \\ Diogo Goulart Corrêa ${ }^{4,5}$
}

Received: 7 December 2021 / Revised: 21 January 2022 / Accepted: 26 January 2022 / Published online: 2 February 2022 (c) ISS 2022

Diagnosis: Hidradenoma.

\section{Imaging interpretation}

Right foot magnetic resonance imaging (MRI) demonstrated an expansile solid-cystic lesion (Fig. 1) in the subcutaneous tissue of the dorsal aspect of the foot. Cystic portions were distinguished as multiple oval areas with hyperintense signal on T1- and fat-suppressed T2-weighted imaging. The cystic portions had thick irregular septations and papillary projections. Solid portions and papillary projections presented gadolinium enhancement, restricted diffusion, and early intense enhancement due to high perfusion (Fig. 2). There were no signs of bone erosion.

The case presentation can be found at doi: $10.1007 / \mathrm{s} 00256-022-$ 04004-w

Fabiana Migliaccio Mansur

fabianammansur92@gmail.com

1 Department of Radiology, Hospital Universitário Clementino Fraga Filho, Federal University of Rio de Janeiro, Rua Rodolpho Paulo Rocco 255, Cidade Universitária, Ilha do Fundão, Rio de Janeiro, RJ 21941-913, Brazil

2 Department of Pathology, Hospital Universitário Clementino Fraga Filho, Federal University of Rio de Janeiro, Rua Rodolpho Paulo Rocco 255, Cidade Universitária, Ilha do Fundão, Rio de Janeiro, RJ 21941-913, Brazil

3 Department of Orthopedics, Hospital Universitário Clementino Fraga Filho, Federal University of Rio de Janeiro, Rua Rodolpho Paulo Rocco 255, Cidade Universitária, Ilha do Fundão, Rio de Janeiro, RJ 21941-913, Brazil

4 Department of Radiology, Clínica de Diagnóstico por Imagem (CDPI)/DASA, Avenida das Américas, 4666 , 302A, 303, 307, 325, 326, Barra da Tijuca, Rio de Janeiro, RJ 2640-102, Brazil

5 Department of Radiology, Hospital Universitário Antônio Pedro, Federal Fluminense University, Rua Marquês de Paraná 303, Centro, Niterói, RJ 24033-900, Brazil
The imaging evaluation led to the differential diagnosis of mycetoma, synovial sarcoma, dermatofibrosarcoma protuberans, and skin adnexa tumor. Because laboratory tests did not show significant alterations, the patient was in good clinical condition, and without skin ulcerations, we considered fungal infection as a remote hypothesis. Conversely, because the lesion was superficial and had been growing slowly for the last 8 years, consistent with a non-aggressive process, and the cystic portions of the lesion presented as hyperintensities in T1-weighted image, probably due to protein content, we considered tumor of skin adnexa origin as the main hypothesis.

After complete surgical removal of the lesion, histopathological analysis revealed a predominantly solid, non-encapsulated dermal tumor with cystic areas. The cystic areas were composed of lobules of cells with clear and eosinophilic cytoplasm immersed in a hyaline stroma, characteristics consistent with hidradenoma (Fig. 3). Currently, the patient is under outpatient follow-up care and has thus far shown no signs of tumor recurrence.

\section{Discussion}

Hidradenoma (a.k.a. clear cell hidradenoma, apocrine hidradenoma, nodular hidradenoma, eccrine acrospiroma, and solid-cystic hidradenoma) is a benign sweat gland tumor with eccrine or apocrine differentiation. It manifests as a well-circumscribed and non-encapsulated dermal nodule, often measuring a few centimeters in diameter, with variable solid and cystic areas. Histopathologically, it is composed mostly of proliferating cells that are pale or clear due to intracellular glycogen. Beyond these pale or clear cells, which led to the alternate name clear cell hidradenoma, other cell types may be present, such as polygonal, mucinous, squamoid, oncocytic, and epidermoid cells, in variable proportions. Typically, the tumor cells have small, round, and eccentrically positioned nuclei. Ductal structures are usually present with associated connective stroma that is 
Fig. 1 Right foot MRI. a T1-weighted image demonstrated that the lesion had predominantly low/intermediate signal intensity, but some cystic portions had hyperintense signal (arrows in a). Fat-suppressed T2-weighted imaging (b) and fat-suppressed proton densityweighted imaging (c) showed multiple thick and irregular septations (arrows) within the lesion. d Gadolinium-enhanced lary projections with enhancement (arrows). The lesion did not appear to be associated with bone erosion image showed septal and papil-
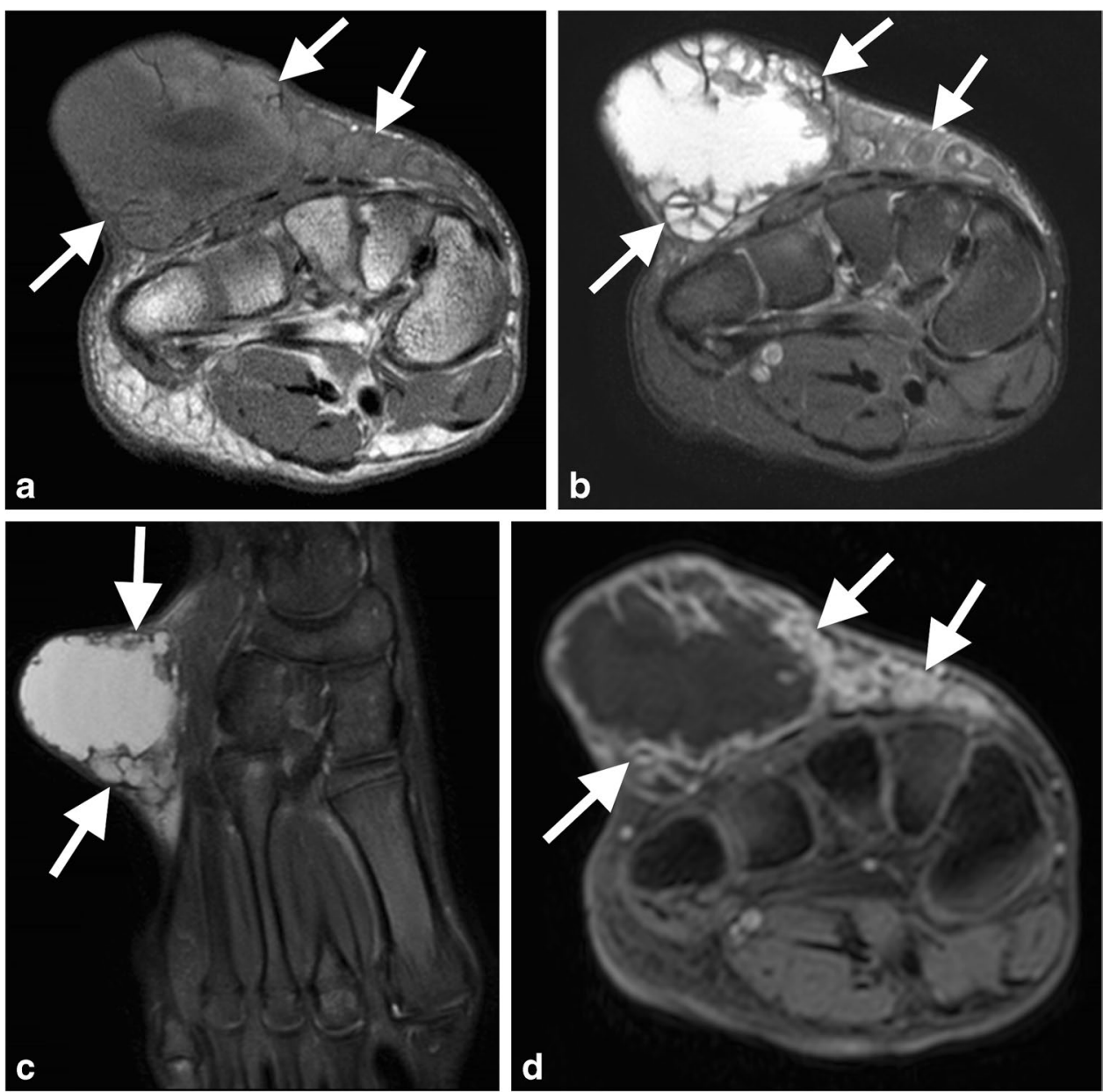

Fig. 2 Advanced MRI. Apparent diffusion coefficient map (a) demonstrated that the solid portions of the lesion had restricted diffusion due to hypercellularity (arrow). Perfusion-weighted image (b) showed areas of early and rapid intense enhancement (arrow) on the color-coded semi-quantitative map
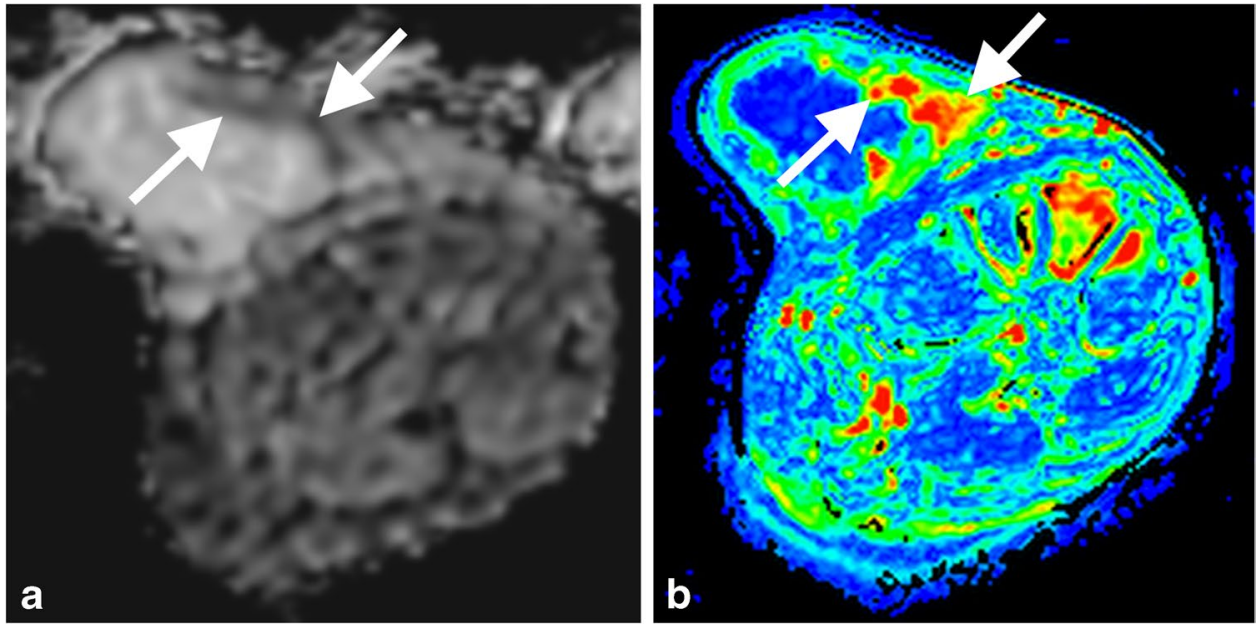

hyaline. The cystic areas are often filled with eosinophilic material [1]. In most cases, immunohistochemical analysis is not necessary for diagnosis.

Hidradenomas have slow and self-limited growth. They are most often found on the head or trunk [2,3]. Although extremities are affected in $24 \%$ of cases, feet are affected in only $3 \%$ of cases [4]. Usually, these tumors appear as solitary lesions covered by normal skin, though ulceration may occur; and they are generally painless $[4,5]$. Demographically, hidradenomas occur most frequently in middleaged patients, and women are affected twice as often as men $[2,3,6]$.

The most characteristic imaging appearance of hidradenomas is a multiloculated cystic mass, with fluid-fluid levels, 
Fig. 3 Histopathology. (a) Hematoxylin and eosin staining (original magnification, $40 \times$ ) demonstrated a nodular dermal tumor with clear cells (blue arrows), cystic spaces (red arrow), and hyaline stroma (green arrow). (b) Hematoxylin and eosin staining in a greater magnification (original magnification, $200 \times$ ) also showing cystic space (red arrow), clear cells (blue arrow), and hyalin stroma (green arrow)
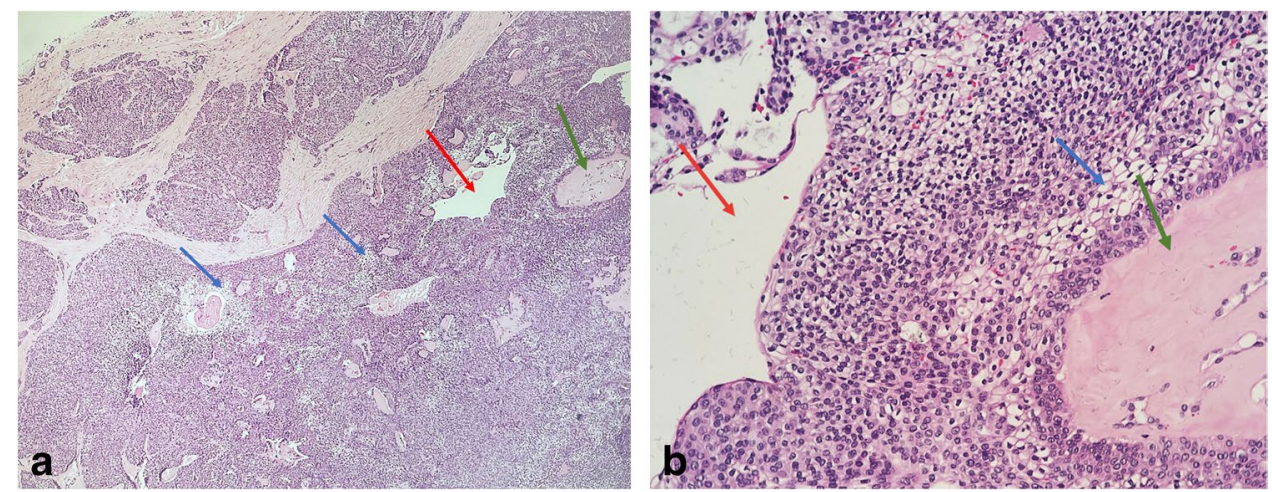

associated with solid mural nodules, with gadoliniumenhancement [4]. The cystic components demonstrate MRI signal intensity variance on $\mathrm{T} 1$ - and $\mathrm{T} 2$-weighted imaging that has been attributed to varying amounts of cholesterol, hemorrhage, and sweat gland secretions [2, 6]. Calcifications may be present. Usually, associated bone erosion and marrow abnormalities are not seen. Rapid growth may indicate malignant conversion [2, 7].

The main differential diagnoses include mycetoma, synovial sarcoma, and dermatofibrosarcoma protuberans. The "dot in a circle sign" refers to multiple conglomerate spherical lesions with hyperintense signal and hypointense rims, with a central hypointense dot on T2-weighted imaging, characteristic of mycetoma [8]. Although the lesion in the present case had multiple cystic components and thick, irregular septations, a central hypointense dot consistent with the "dot in a circle sign" was not observed on T2-weighted imaging. Usually, synovial sarcoma presents as a deep seated and heterogeneous soft tissue mass with hemorrhage, fluid levels, and septa, aspects that together create the "triple sign" [6, 9]. Calcification can be seen in up to $30 \%$ of synovial sarcomas in radiographs [9]. Although our patient did not perform radiography or computed tomography, for a better assessment of the presence of calcifications, the lesion was superficial, and did not cause bone erosion. A superficial soft-tissue mass, with homogenous isointense signal on T1-, hyperintense signal on T2-weighted imaging, and homogeneous enhancement should suggest dermatofibrosarcoma protuberans [10], whereas our patient had a heterogeneous lesion. Also, dermatofibrosarcoma protuberans is more common in the trunk and head and neck, and very rare in the inferior extremities [10].

Although histopathological analysis is essential for hidradenoma diagnosis, conventional MRI can contribute to its diagnosis consideration, particularly when evaluating a superficial slow-growing, solid, and cystic lesion without evident bone erosion. MRI can reveal the glandular component of a hidradenoma, which appears as a hyperintense signal on T1-weighted imaging due to its hemorrhagic or protein content. While advanced MRI sequences may show signs of tumor activity, such as diffusion restriction or hyperperfusion, they can also reveal clues for biopsy targeting and they should not confound the differential diagnosis.

\section{Declarations}

Ethics approval All procedures performed in studies involving human participants were in accordance with ethical standards of the institutional and national research committee and with the 1964 Helsinki declaration and its later amendments or comparable ethical standards.

Informed consent Informed consent was obtained from the participant included in the study.

Animal rights This article does not contain any studies with animal subjects performed by the any of the authors.

Conflict of interest The authors declare no competing interests.

\section{References}

1. Patterson JW. Weedon's skin pathology. 5th ed. London, United Kingdom: Elsevier Limited; 2019. p. 951-1051.

2. Gandhi D, Ahuja K, Nepal P, Sawhney H, Sharma P, Kier R. Clear cell hidradenoma of the foot: a case report with literature review. AME Med J. 2020;5:33.

3. Suleimenova DM, Ojeda-Fournier H. Multimodality imaging of nodulocystic hidradenoma of the breast. J Breast Imaging. 2019;1(4):358-9.

4. Mulcahy H. Big foot MRI: a practical guide for radiologists to soft tissue tumors and tumor-like lesions of the foot. Semin Roentgenol. 2019;54(2):162-76.

5. Feldman AH, Niemi WJ, Blume PA, Chaney DM. Clear cell hidradenoma of the second digit: a review of the literature with case presentation. J Foot Ankle Surg. 1997;36(1):21-3.

6. Lee JY, Kang BS, Shim HS, et al. Clear cell hidradenoma: characteristic imaging features on ultrasonography, computed tomography, and magnetic resonance imaging. J Ultrasound Med. 2018;37(8):1993-2001.

7. Mullaney PJ, Becker E, Graham B, Ghazarian D, Riddell RH, Salonen DC. Benign hidradenoma: magnetic resonance and ultrasound features of two cases. Skeletal Radiol. 2007;36(12):1185-90. 
8. Neelakantan S, Babu AA, Anandarajan R. 'Dot in circle sign': a characteristic finding in ultrasound and MR imaging of soft tissue mycetomas. BMJ Case Rep. 2016;2016:bcr2016216502.

9. Murphey MD, Gibson MS, Jennings BT, Crespo-Rodríguez AM, Fanburg-Smith J, Gajewski DA. From the archives of the AFIP: Imaging of synovial sarcoma with radiologic-pathologic correlation. Radiographics. 2006;26(5):1543-65.

10. Zhang L, Liu Q, Cao Y, Zhong J, Zhang W. Dermatofibrosarcoma protuberans: computed tomography and magnetic resonance imaging findings. Medicine. 2015;94(24):1001.
Publisher's note Springer Nature remains neutral with regard to jurisdictional claims in published maps and institutional affiliations. 